\title{
The Topological Variable Computation for a Special Type of Cycloalkanes
}

\author{
Wei Gao, ${ }^{1,2}$ Yaojun Chen, ${ }^{1}$ and Weifan Wang ${ }^{3}$ \\ ${ }^{1}$ Department of Mathematics, Nanjing University, Nanjing 210093, China \\ ${ }^{2}$ School of Information Science and Technology, Yunnan Normal University, Kunming 650500, China \\ ${ }^{3}$ Department of Mathematics, Zhejiang Normal University, Jinhua 321004, China \\ Correspondence should be addressed to Yaojun Chen; yaojunc@nju.edu.cn
}

Received 14 May 2017; Accepted 28 June 2017; Published 22 August 2017

Academic Editor: Tomokazu Yoshimura

Copyright (C) 2017 Wei Gao et al. This is an open access article distributed under the Creative Commons Attribution License, which permits unrestricted use, distribution, and reproduction in any medium, provided the original work is properly cited.

\begin{abstract}
As an efficient theoretical tool, graph theory is widely used in computing chemistry. In terms of index computation on molecular graphs, the researchers can learn the potential properties of chemical compounds, including drugs, materials, and organics. In this paper, by means of distance computation, we study the eccentric version indices of cycloalkanes which occur quite frequently in the chemical drugs and other compounds. The promising prospects of the application for the physical, chemical, medical, and pharmacy engineering are illustrated by theoretical conclusions obtained in this article.
\end{abstract}

\section{Introduction}

In the early years, followed by the chemical experiments, the scientists found that there is a close relationship between characterizations of chemical compounds and their molecular structures (e.g., Wiener index reflects both the melting point and boiling point of chemical compounds; see Wiener [1] and Katritzky et al. [2]). This discovery inspired researchers to study the feature of drugs, materials, and compounds by analyzing their molecular structures. The computational model can be expressed as follows: we use a graph (such graph is called molecular graph) to represent each molecular structure where each vertex denotes an atom and each edge represents a chemical bond between atoms; then we define the topological indices on molecular graphs where each index reflects a certain chemical character of compound; at last, by computing these topological indices, we estimate the chemical, biology, medicine, and pharmacology features of compounds. In this way, as a branch of theoretical chemistry, topological index calculation becomes a hot topic among the scholars from different research files.

Specifically, let $G=(V(G), E(G))$ be a molecular graph with vertex (atom) set $V(G)$ and edge (chemical bond) set $E(G)$, and the topological index can be considered as a score function $f: G \rightarrow \mathbb{R}^{+}$which maps each molecular graph to a positive real number. These defined topological indices are functioned as numerical descriptors of the molecular structure yielded from the corresponding chemical compound, and many engineering applications of it can be implemented in theoretical chemistry, especially in QSPR/QSAR study. For example, scholars introduce PI index, Wiener index, harmonic index, atom-bond connectivity index, Shultz index, and Gutman index to reflect certain structural features of molecules. There were several articles contributing to determine these distance-based indices of special molecular graph (see Gao and Siddiqui [3], Zhao and Wu [4], Gao et al. [5-9], Hosamani et al. [10], Gao and Wang [11, 12], and Basavanagoud et al. [13] for more details). The notation and terminology that were used but undefined in this paper can be found in [14].

The eccentricity ec $(u)$ of vertex $u \in V(G)$ is defined as the maximum distance between $u$ and any other vertex in $G$. The fifth atom-bond connectivity index (eccentricity version of atom-bond connectivity index) of molecular graph $G$ is defined as

$$
A B C_{5}(G)=\sum_{u v \in E(G)} \sqrt{\frac{\mathrm{ec}(u)+\mathrm{ec}(v)-2}{\mathrm{ec}(u) \mathrm{ec}(v)}} .
$$


The first multiplicative Zagreb index and the second multiplicative Zagreb index are denoted by

$$
\begin{aligned}
& \pi_{1}^{*}(G)=\prod_{v \in V(G)}(\mathrm{ec}(v))^{2}, \\
& \pi_{2}^{*}(G)=\prod_{u v \in E(G)}(\mathrm{ec}(u) \mathrm{ec}(v)),
\end{aligned}
$$

respectively.

The fourth geometric-arithmetic index is denoted by

$$
G A_{4}(G)=\sum_{u v \in E(G)} \frac{2 \sqrt{\mathrm{ec}(u) \mathrm{ec}(v)}}{\mathrm{ec}(u)+\mathrm{ec}(v)}
$$

The fourth Zagreb index was formulated as

$$
Z g_{4}(G)=\sum_{u v \in E(G)}(\mathrm{ec}(u)+\mathrm{ec}(v)) .
$$

The fifth multiplicative atom bond connectivity index is stated as

$$
A B C \Pi_{5}(G)=\prod_{u v \in E(G)} \sqrt{\frac{\mathrm{ec}(u)+\mathrm{ec}(v)-2}{\mathrm{ec}(u) \mathrm{ec}(v)}} .
$$

More eccentricity version topological indices and their theoretical results can refer to López-Candales et al. $[15,16]$, Vukičević and Graovac [17], Das et al. [18], Mouratoglou et al. [19], Farooq and Malik [20], Farooq et al. [21], McCrary et al. [22], Abraham and Weismann [23], Yamasaki et al. [24], Guirao and de Bustos [25], Dimitrov [26-28], and Ahmadi et al. [29].

Cycloalkane is a kind of saturated hydrocarbon containing an alicyclic structure in organic chemistry and it contains an alicyclic ring and unsubstituted alkyl on the ring. To explain it more clearly, a cycloalkane is made up with nothing but hydrogen and carbon atoms which are distributed randomly in a system with only a single ring and single carbon-carbon bonds. Totally, four types of cycloalkanes are classified by researchers, which are small, common, medium, and large. The general formula of it is denoted as $C_{n} H_{2(n+1-r)}$, where $n$ is used to denote the number of carbon atoms and $r$ to denote that of rings. Naphthene is used to describe the group of cycloalkanes. The main source of naphthenes is petroleum and natural gas. In addition, cycloalkanes are alicyclic hydrocarbons in the petroleum industry. After millions of years of decay and the geological stress, the complex organic compounds of animals and plants have become a mixture of alkanes and naphthenes whose size varies from 1 carbon to 30 or 40 carbons.

Basically speaking, cycloalkanes share similar general physical properties and attributes with alkanes. However, it possesses some unique or different properties. To illustrate, the boiling point and melting point of cycloalkanes are higher than the common alkanes. Besides, it has a high calorific value and a low freezing point. Its antiknock quality is between that of the normal hydrocarbon and the isomeric hydrocarbon.

As described above, there are four types of cycloalkanes, and the bigger cycloalkanes are quite stable. For instance, the

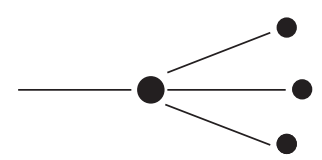

FIGURE 1: The molecular structure of $R_{1}$ : meythl.

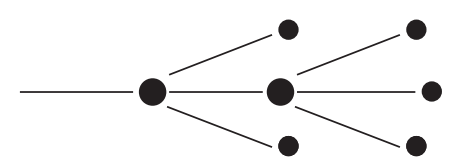

FIgURE 2: The molecular structure of $R_{2}$ : eythl.

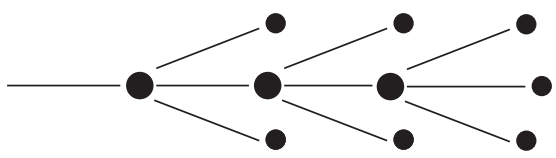

Figure 3: The molecular structure of $R_{3}$ : propyl.

radical chain reactions of the alkanes are pretty stable. Macrocyclic alkanes do not react with strong acids, strong bases, strong oxidants, and so on. Conversely, thanks to Baeyer strain and ring strain, the stability of small cycloalkanes tend to be much lower, among which, cyclopropane is one of the most typical examples. They do not react in electrophilic addition but react in nucleophilic aliphatic substitution. At room temperature, cycloalkanes do not work with general oxidants (such as potassium permanganate solution and ozone), even with cyclopropane. If the oxidation possesses a strong oxidant or, under the action of the catalyst with air oxidation, cycloalkanes can also have an oxidation reaction. For example, adipic acid is the raw material for the synthesis of nylon, which is the main material for clothing.

The effect of cycloalkane is mainly to be used to produce fuel in the applications. In naphthenes, what is the most popular cycloalkanes applied in the industry may be cyclohexane, a colorless liquid, which is easy to volatile and burn. Natural gas and biogas contain a lot of methane, which has been used extensively in recent years as clean energy, and it is quite popular nowadays. Hence, it can also be used in lubricants and asphalt. Besides, as an important chemical raw material, cycloalkanes have been largely used in medicine field and alcohols are traditionally considered as the main ingredient of many important medicines.

More contexts on cycloalkanes and its applications can refer to El-Sayed et al. [30], Vásquez-Espinal et al. [31], Caroen et al. [32], Denicourt-Nowicki et al. [33], El-Gamal et al. [34, 35], Lepori et al. [36], Nambu et al. [37], Lazić et al. [38], Alaoui et al. [39], and Pi-Boleda et al. [40]. Since cycloalkanes commonly appeared in the chemical compound and possess wide engineering applications in the field of biology, medicine, and pharmacy, it inspires us to study the characterization of cycloalkanes from mathematical point of view.

Let $R_{1}$ (meythl), $R_{2}$ (eythl), $R_{3}$ (propyl), .., $R_{r}$ be the group of alkyls (the molecular structures can refer to Figures $1,2,3$, and 4 for more details). 


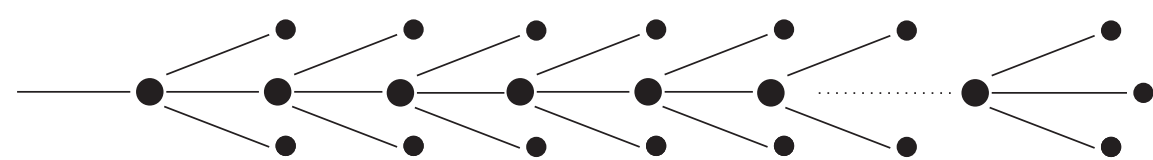

Figure 4: The molecular structure of $R_{i}$ : alkyl.

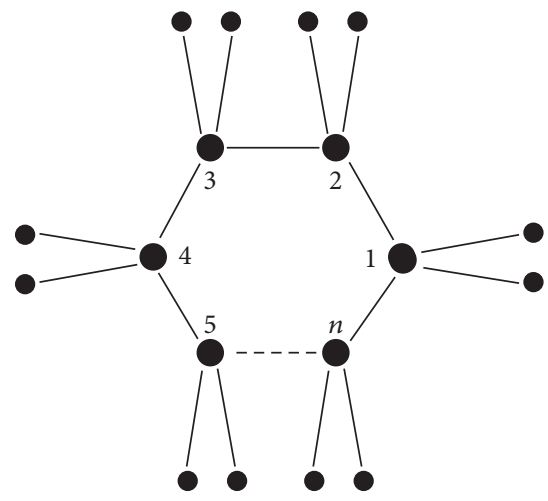

FIGURE 5: The molecular structure of cycloalkanes $C_{n}^{2}$.

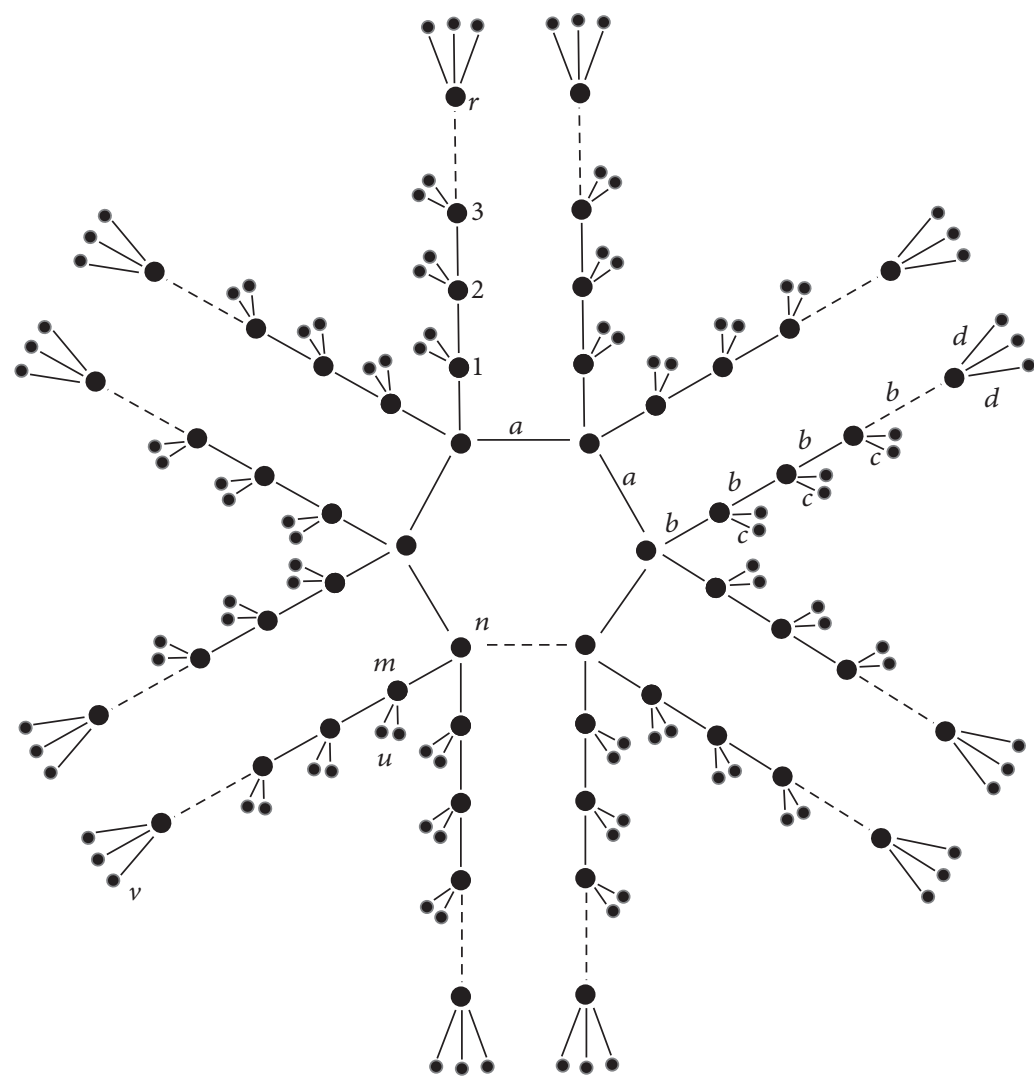

FIGURE 6: Molecular graph representing the chemical compound of $C_{n}^{R_{i}}$.

In the following section, we discuss certain topological indices of a special kind of cycloalkanes, and its molecular graphs are constructed by attaching alkyl $R_{i}$ instead of each hydrogen atoms in cycloalkanes structures. Let $C_{n}^{2}$ be the cycloalkanes which are yielded by attaching two hydrogen atoms to each carbon atoms (see Figure 5). Moreover, let $C_{n}^{R_{i}}$ be molecular graph constructed by attaching $R_{i}$ instead of each hydrogen atom in cycloalkanes which are presented in Figure 6.

The main purpose of this article is to obtain certain eccentricity version topological indices of this family of cycloalkanes $C_{n}^{R_{i}}$. 


\section{Main Results and Proofs}

In this section, we present the main conclusions of our paper which provide theoretical support for future engineering applications. In what follows, we always assume that $n$ and $r$ are both positive integers. Furthermore, $n>2$ if $n \equiv$ $1(\bmod 2)$ and $n>3$ if $n \equiv 0(\bmod 2)$.

The first result stated as follows reveals the first multiplicative Zagreb index of cycloalkanes.

Theorem 1. The first multiplicative Zagreb index of $C_{n}^{R_{i}}$ is

$$
\begin{aligned}
\pi_{1}^{*}\left(C_{n}^{R_{i}}\right) \\
= \begin{cases}\left(\frac{n}{2}+r+1\right)^{2 n} \prod_{i=1}^{r}\left(\frac{n}{2}+r+i+1\right)^{4 n} \prod_{i=2}^{r}\left(\frac{n}{2}+r+i+1\right)^{8 n} \cdot\left(\frac{n}{2}+2 r+2\right)^{12 n}, & \text { if } n \equiv 0(\bmod 2), \\
\left(\frac{n-1}{2}+r+1\right)^{2 n} \prod_{i=1}^{r}\left(\frac{n-1}{2}+r+i+1\right)^{4 n} \prod_{i=2}^{r}\left(\frac{n-1}{2}+r+i+1\right)^{8 n} \cdot\left(\frac{n-1}{2}+2 r+2\right)^{12 n}, & \text { if } n \equiv 1(\bmod 2) .\end{cases}
\end{aligned}
$$

Proof of Theorem 1. We discuss the following two cases according to the parity of $n$.

Case $1(n \equiv 0(\bmod 2))$. In this case, the vertex (atom) set of $C_{n}^{R_{i}}$ can be divided into the following four classes:

(i) ec $(v)=n / 2+r+1$, and there are $n$ such vertices.

(ii) For $i \in\{1, \ldots, r\}, \mathrm{ec}(v)=n / 2+r+i+1$, and there are $2 n$ such vertices in each subclass;

(iii) For $i \in\{2, \ldots, r\}$, ec $(v)=n / 2+r+i+1$, and there are $4 n$ such vertices in each subclass.

(iv) $\mathrm{ec}(v)=n / 2+2 r+2$, and there are $6 n$ such vertices.

Thus, in light of the definition of the first multiplicative Zagreb index, we infer

$$
\begin{aligned}
& \pi_{1}^{*}\left(C_{n}^{R_{i}}\right)=\prod_{v \in V\left(C_{n}^{R_{i}}\right)}(\mathrm{ec}(v))^{2}=\left(\frac{n}{2}+r+1\right)^{2 n} \\
& \cdot \prod_{i=1}^{r}\left(\frac{n}{2}+r+i+1\right)^{4 n} \prod_{i=2}^{r}\left(\frac{n}{2}+r+i+1\right)^{8 n} \\
& \cdot\left(\frac{n}{2}+2 r+2\right)^{12 n} .
\end{aligned}
$$

Case $2(n \equiv 1(\bmod 2))$. In this case, the vertex (atom) set of $C_{n}^{R_{i}}$ can be separated into the following four classes: (i) ec $(v)=(n-1) / 2+r+1$, and there are $n$ such vertices.

(ii) For $i \in\{1, \ldots, r\}$, ec $(v)=(n-1) / 2+r+i+1$, and there are $2 n$ such vertices in each subclass.

(iii) For $i \in\{2, \ldots, r\}, \mathrm{ec}(v)=(n-1) / 2+r+i+1$, and there are $4 n$ such vertices in each subclass.

(iv) ec $(v)=(n-1) / 2+2 r+2$, and there are $6 n$ such vertices.

Hence, in view of the definition of the first multiplicative Zagreb index, we deduce

$$
\begin{aligned}
\pi_{1}^{*} & \left(C_{n}^{R_{i}}\right)=\prod_{v \in V\left(C_{n}^{R_{i}}\right)}(\mathrm{ec}(v))^{2}=\left(\frac{n-1}{2}+r+1\right)^{2 n} \\
\cdot & \prod_{i=1}^{r}\left(\frac{n-1}{2}+r+i+1\right)^{4 n} \prod_{i=2}^{r}\left(\frac{n-1}{2}+r+i+1\right)^{8 n} \\
& \cdot\left(\frac{n-1}{2}+2 r+2\right)^{12 n} .
\end{aligned}
$$

Therefore, we get the desired result.

The next result stated as follows implies the second multiplicative Zagreb index of cycloalkanes.

Theorem 2. The second multiplicative Zagreb index of $C_{n}^{R_{i}}$ is

$$
\begin{aligned}
& \pi_{2}^{*}\left(C_{n}^{R_{i}}\right) \\
& = \begin{cases}\left(\frac{n}{2}+r+1\right) \prod_{i=0}^{2 n} \prod^{r-1}\left(\left(\frac{n}{2}+r+i+1\right)\left(\frac{n}{2}+r+i+2\right)\right)^{2 n} \cdot \prod_{i=1}^{r-1}\left(\left(\frac{n}{2}+r+i+1\right)\left(\frac{n}{2}+r+i+2\right)\right)^{4 n} \cdot\left(\left(\frac{n}{2}+2 r+1\right)\left(\frac{n}{2}+2 r+2\right)\right)^{6 n}, & \text { if } n \equiv 0(\bmod 2), \\
\left(\frac{n-1}{2}+r+1\right)^{2 n} \prod_{i=0}^{r-1}\left(\left(\frac{n-1}{2}+r+i+1\right)\left(\frac{n-1}{2}+r+i+2\right)\right)^{2 n} \cdot \prod_{i=1}^{r-1}\left(\left(\frac{n-1}{2}+r+i+1\right)\left(\frac{n-1}{2}+r+i+2\right)\right)^{4 n} & \text { if } n \equiv 1(\bmod 2) . \\
\cdot\left(\left(\frac{n-1}{2}+2 r+1\right)\left(\frac{n-1}{2}+2 r+2\right)\right)^{6 n}, & \end{cases}
\end{aligned}
$$


Proof of Theorem 2. The proof procedure can be divided into the following two cases in terms of the parity of $n$.

Case $1(n \equiv 0(\bmod 2))$. In this case, the edge (chemical bond) set of $C_{n}^{R_{i}}$ can be divided into the following four classes:

(i) $(\mathrm{ec}(u), \mathrm{ec}(v))=(n / 2+r+1, n / 2+r+1)$, and there are $n$ edges in this class.

(ii) For $i \in\{0, \ldots, r-1\},(\mathrm{ec}(u), \mathrm{ec}(v))=(n / 2+r+i+$ $1, n / 2+r+i+2)$, and there are $2 n$ edges in each subclass.

(iii) For $i \in\{1, \ldots, r-1\},(\mathrm{ec}(u), \mathrm{ec}(v))=(n / 2+r+i+$ $1, n / 2+r+i+2)$, and there are $4 n$ edges in each subclass.

(iv) $(\mathrm{ec}(u), \mathrm{ec}(v))=(n / 2+2 r+1, n / 2+2 r+2)$, and there are $6 n$ edges in this class.

Then, by means of the definition of the second multiplicative Zagreb index, we have

$$
\begin{aligned}
\pi_{2}^{*}\left(C_{n}^{R_{i}}\right)=\prod_{u v \in E\left(C_{n}^{R_{i}}\right)}(\mathrm{ec}(u) \mathrm{ec}(v))=\left(\frac{n}{2}+r+1\right)^{2 n} \\
\cdot \prod_{i=0}^{r-1}\left(\left(\frac{n}{2}+r+i+1\right)\left(\frac{n}{2}+r+i+2\right)\right)^{2 n} \\
\cdot \prod_{i=1}^{r-1}\left(\left(\frac{n}{2}+r+i+1\right)\left(\frac{n}{2}+r+i+2\right)\right)^{4 n} \\
.\left(\left(\frac{n}{2}+2 r+1\right)\left(\frac{n}{2}+2 r+2\right)\right)^{6 n} .
\end{aligned}
$$

Case $2(n \equiv 1(\bmod 2))$. In this case, the edge (chemical bond) set of $C_{n}^{R_{i}}$ can be separated into the following four classes:

(i) $(\mathrm{ec}(u), \mathrm{ec}(v))=((n-1) / 2+r+1,(n-1) / 2+r+1)$, and there are $n$ edges in this class.

(ii) For $i \in\{0, \ldots, r-1\},(\mathrm{ec}(u), \mathrm{ec}(v))=((n-1) / 2+r+$ $i+1,(n-1) / 2+r+i+2)$, and there are $2 n$ edges in each subclass.

(iii) For $i \in\{1, \ldots, r-1\},(\mathrm{ec}(u), \mathrm{ec}(v))=((n-1) / 2+r+$ $i+1,(n-1) / 2+r+i+2)$, and there are $4 n$ edges in each subclass.

(iv) $(\mathrm{ec}(u), \mathrm{ec}(v))=((n-1) / 2+2 r+1,(n-1) / 2+2 r+2)$, and there are $6 n$ edges in this class.

Using the definition of the second multiplicative Zagreb index, we get

$$
\begin{aligned}
\pi_{2}^{*} & \left(C_{n}^{R_{i}}\right)=\prod_{u v \in E\left(C_{n}^{R_{i}}\right)}(\mathrm{ec}(u) \mathrm{ec}(v)) \\
& =\left(\frac{n-1}{2}+r+1\right)^{2 n} \\
& \cdot \prod_{i=0}^{r-1}\left(\left(\frac{n-1}{2}+r+i+1\right)\left(\frac{n-1}{2}+r+i+2\right)\right)^{2 n} \\
& \cdot \prod_{i=1}^{r-1}\left(\left(\frac{n-1}{2}+r+i+1\right)\left(\frac{n-1}{2}+r+i+2\right)\right)^{4 n} \\
& \cdot\left(\left(\frac{n-1}{2}+2 r+1\right)\left(\frac{n-1}{2}+2 r+2\right)\right)^{6 n} .
\end{aligned}
$$

Finally, we obtain the desired result.
Theorem 3. The fourth Zagreb index of $C_{n}^{R_{i}}$ is

$$
\begin{aligned}
& Z g_{4}(G)\left(C_{n}^{R_{i}}\right) \\
& = \begin{cases}6 n^{2} r+3 n^{2}+18 n r^{2}+30 n r+8 n, & \text { if } n \equiv 0(\bmod 2), \\
6 n^{2} r+3 n^{2}+18 n r^{2}+24 n r+5 n, & \text { if } n \equiv 1(\bmod 2) .\end{cases}
\end{aligned}
$$

Proof of Theorem 3. If $n$ is even, then

$$
\begin{aligned}
Z g_{4}( & \left.C_{n}^{R_{i}}\right)=\sum_{u v \in E\left(C_{n}^{R_{i}}\right)}(\mathrm{ec}(u)+\mathrm{ec}(v)) \\
= & 2 n\left(\frac{n}{2}+r+1\right) \\
& +\sum_{i=0}^{r-1} 2 n\left(\left(\frac{n}{2}+r+i+1\right)+\left(\frac{n}{2}+r+i+2\right)\right) \\
& +\sum_{i=1}^{r-1} 4 n\left(\left(\frac{n}{2}+r+i+1\right)+\left(\frac{n}{2}+r+i+2\right)\right) \\
& +6 n\left(\left(\frac{n}{2}+2 r+1\right)+\left(\frac{n}{2}+2 r+2\right)\right) \\
= & 6 n^{2} r+3 n^{2}+18 n r^{2}+30 n r+8 n .
\end{aligned}
$$

If $n$ is odd, then

$$
\begin{aligned}
& Z g_{4}\left(C_{n}^{R_{i}}\right)=\sum_{u v \in E\left(C_{n}^{R_{i}}\right)}(\mathrm{ec}(u)+\mathrm{ec}(v))=2 n\left(\frac{n-1}{2}+r\right. \\
& +1)+\sum_{i=0}^{r-1} 2 n\left(\left(\frac{n-1}{2}+r+i+1\right)\right. \\
& \left.+\left(\frac{n-1}{2}+r+i+2\right)\right) \\
& +\sum_{i=1}^{r-1} 4 n\left(\left(\frac{n-1}{2}+r+i+1\right)\right. \\
& \left.+\left(\frac{n-1}{2}+r+i+2\right)\right)+6 n\left(\left(\frac{n-1}{2}+2 r+1\right)\right. \\
& \left.+\left(\frac{n-1}{2}+2 r+2\right)\right)=6 n^{2} r+3 n^{2}+18 n r^{2}+24 n r \\
& +5 n .
\end{aligned}
$$

The desired conclusion is proofed.

Followed by the trick and intermediate result presented in Theorems 2 and 3, we derive the following results.

Theorem 4. The fifth atom-bond connectivity index of $C_{n}^{R_{i}}$ is 


$$
\begin{aligned}
& A B C_{5}\left(C_{n}^{R_{i}}\right) \\
& = \begin{cases}\frac{n \sqrt{n+2 r}}{n / 2+r+1}+\sum_{i=0}^{r-1} 2 n \sqrt{\frac{n+2 r+2 i+1}{(n / 2+r+i+1)(n / 2+r+i+2)}}+\sum_{i=1}^{r-1} 4 n \sqrt{\frac{n+2 r+2 i+1}{(n / 2+r+i+1)(n / 2+r+i+2)}}+6 n \sqrt{\frac{n+4 r+1}{(n / 2+2 r+1)(n / 2+2 r+2)}}, & \text { if } n \equiv 0(\bmod 2) \\
\frac{n \sqrt{n+2 r-1}}{(n-1) / 2+r+1}+\sum_{i=0}^{r-1} 2 n \sqrt{\frac{n+2 r+2 i}{((n-1) / 2+r+i+1)((n-1) / 2+r+i+2)}}+\sum_{i=1}^{r-1} 4 n \sqrt{\frac{n+2 r+2 i}{((n-1) / 2+r+i+1)((n-1) / 2+r+i+2)}} \\
+6 n \sqrt{\frac{n+4 r}{((n-1) / 2+2 r+1)((n-1) / 2+2 r+2)}}, & \text { if } n \equiv 1(\bmod 2) .\end{cases}
\end{aligned}
$$

Theorem 5. The fourth geometric-arithmetic index of $C_{n}^{R_{i}}$ is

$$
\begin{aligned}
& G A_{4}\left(C_{n}^{R_{i}}\right) \\
& = \begin{cases}n+\sum_{i=0}^{r-1} \frac{4 n \sqrt{(n / 2+r+i+1)(n / 2+r+i+2)}}{n+2 r+2 i+3}+\sum_{i=1}^{r-1} \frac{8 n \sqrt{(n / 2+r+i+1)(n / 2+r+i+2)}}{n+2 r+2 i+3}+\frac{12 n \sqrt{(n / 2+2 r+1)(n / 2+2 r+2)}}{n+4 r+3}, & \text { if } n \equiv 0(\bmod 2), \\
n+\sum_{i=0}^{r-1} \frac{4 n \sqrt{((n-1) / 2+r+i+1)((n-1) / 2+r+i+2)}}{n+2 r+2 i+2}+\sum_{i=1}^{r-1} \frac{8 n \sqrt{((n-1) / 2+r+i+1)((n-1) / 2+r+i+2)}}{n+2 r+2 i+2} & \text { if } n \equiv 1(\bmod 2) . \\
+\frac{12 n \sqrt{((n-1) / 2+2 r+1)((n-1) / 2+2 r+2)}}{n+4 r+2}, & \end{cases}
\end{aligned}
$$

Theorem 6. The fifth multiplicative atom-bond connectivity index of $C_{n}^{R_{i}}$ is

$$
\begin{aligned}
& A B C \Pi_{5}\left(C_{n}^{R_{i}}\right) \\
& = \begin{cases}\left(\frac{\sqrt{n+2 r}}{n / 2+r+1}\right)^{n} \prod_{i=0}^{r-1}\left(\sqrt{\frac{n+2 r+2 i+1}{(n / 2+r+i+1)(n / 2+r+i+2)}}\right)^{2 n} \cdot \prod_{i=1}^{r-1}\left(\sqrt{\frac{n+2 r+2 i+1}{(n / 2+r+i+1)(n / 2+r+i+2)}}\right)^{4 n} \cdot\left(\sqrt{\frac{n+4 r+1}{(n / 2+2 r+1)(n / 2+2 r+2)}}\right)^{6 n}, & \text { if } n \equiv 0(\bmod 2), \\
\left(\frac{\sqrt{n+2 r-1}}{(n-1) / 2+r+1}\right)^{n} \prod_{i=0}^{n-1}\left(\sqrt{\frac{n+2 r+2 i}{((n-1) / 2+r+i+1)((n-1) / 2+r+i+2)}}\right)^{2 n} \cdot \prod_{i=1}^{r-1}\left(\sqrt{\frac{n+2 r+2 i}{((n-1) / 2+r+i+1)((n-1) / 2+r+i+2)}}\right)^{4 n} \\
\cdot\left(\sqrt{\frac{n+4 r}{((n-1) / 2+2 r+1)((n-1) / 2+2 r+2)}}\right)^{6 n}, & \text { if } n \equiv 1(\bmod 2) .\end{cases}
\end{aligned}
$$

\section{Conclusion}

In this paper, we report several important topological indices of cycloalkanes $C_{n}^{R_{i}}$ with the help of graph theory and mathematical derivation. The atom-bond connectivity indices, geometric-arithmetic indices, and Zagreb indices are widely used in the analysis of melting (boiling) point and other chemical characters for chemical compounds and QSPR/QSAR study. Hence, the promising prospects of the application for the material, chemical, and pharmacy engineering will be illustrated in the theoretical conclusions that are yielded in our article.

The molecular graph $C_{n}^{R_{i}}$ is a way to obtain chemical structure by using cycloalkanes to attach alkyl $R_{i}$ instead of each hydrogen atom. Similarly, more chemical structures can be yielded by replacing alkyl $R_{i}$ with other structures. That is to say, this trick has potential application prospects in chemistry to deal with various commonly appeared chemical structures.

\section{Conflicts of Interest}

The authors declare that there are no conflicts of interest regarding the publication of this paper.

\section{Acknowledgments}

The authors are grateful to Dr. Raad Sehen Haoer for his valuable comments on the manuscript. The research is 
partially supported by NSFC (nos. 11401519, 11371193, and 11671198).

\section{References}

[1] H. Wiener, "Structural determination of paraffin boiling points," Journal of the American Chemical Society, vol. 69, no. 1, pp. 1720, 1947

[2] A. R. Katritzky, R. Jain, A. Lomaka, R. Petrukhin, U. Maran, and M. Karelson, "Perspective on the relationship between melting points and chemical structure," Crystal Growth and Design, vol. 1, no. 4, pp. 261-265, 2001.

[3] W. Gao and M. K. Siddiqui, "Molecular descriptors of nanotube, oxide, silicate, and triangulene networks," Journal of Chemistry, vol. 2017, 10 pages, 2017.

[4] B. Zhao and H. L. Wu, "Pharmacological characteristics analysis of two molecular structures," Applied Mathematics and Nonlinear Sciences, vol. 2, no. 1, pp. 93-110, 2017.

[5] W. Gao, Y. Wang, W. Wang, and L. Shi, "The first multiplication atom-bond connectivity index of molecular structures in drugs," Saudi Pharmaceutical Journal, vol. 25, no. 4, pp. 548555, 2017.

[6] W. Gao, L. Yan, and L. Shi, "Generalized Zagreb index of polyomino chains and nanotubes," Optoelectronics and Advanced Materials-Rapid Communications, vol. 11, no. 1-2, pp. 119-124, 2017.

[7] W. Gao, W. Wang, M. K. Jamil, and M. R. Farahani, "Electron energy studying of molecular structures via forgotten topological index computation," Journal of Chemistry, vol. 2016, 7 pages, 2016.

[8] W. Gao, M. K. Siddiqui, M. Imran, M. K. Jamil, and M. R. Farahani, "Forgotten topological index of chemical structure in drugs," Saudi Pharmaceutical Journal, vol. 24, no. 3, pp. 258264, 2016.

[9] W. Gao, W. Wang, and M. R. Farahani, “Topological indices study of molecular structure in anticancer drugs," Journal of Chemistry, vol. 2016, Article ID 3216327, 8 pages, 2016.

[10] S. M. Hosamani, B. B. Kulkarni, R. G. Boli, and V. M. Gadag, "QSPR analysis of certain graph theocratical matrices and their corresponding energy," Applied Mathematics and Nonlinear Sciences, vol. 2, no. 1, pp. 131-150, 2017.

[11] W. Gao and W. F. Wang, "The fifth geometric-arithmetic index of bridge graph and carbon nanocones," Journal of Difference Equations and Applications, vol. 23, no. 1-2, pp. 100-109, 2017.

[12] W. Gao and W. Wang, "The eccentric connectivity polynomial of two classes of nanotubes," Chaos, Solitons and Fractals, vol. 89, pp. 290-294, 2016.

[13] B. Basavanagoud, V. R. Desai, and S. Patil, " $(\beta, \alpha)$ connectivity index of graphs," Applied Mathematics and Nonlinear Sciences, vol. 2, no. 1, pp. 21-30, 2017.

[14] J. A. Bondy and U. S. R. Murty, Graph Theory, Springer, Berlin, Germany, 2008.

[15] A. López-Candales, N. Rajagopalan, M. Kochar, B. Gulyasy, and K. Edelman, "Systolic eccentricity index identifies right ventricular dysfunction in pulmonary hypertension," International Journal of Cardiology, vol. 129, no. 3, pp. 424-426, 2008.

[16] A. López-Candales, R. Bazaz, K. Edelman, and B. Gulyasy, "Apical systolic eccentricity index: A better marker of right ventricular compromise in pulmonary hypertension," Echocardiography, vol. 27, no. 5, pp. 534-538, 2010.
[17] D. Vukičević and A. Graovac, "Note on the comparison of the first and second normalized Zagreb eccentricity indices," Acta Chimica Slovenica, vol. 57, no. 3, pp. 524-528, 2010.

[18] K. C. Das, D.-W. Lee, and A. Graovac, "Some properties of the Zagreb eccentricity indices," Ars Mathematica Contemporanea, vol. 6, no. 1, pp. 117-125, 2013.

[19] S. A. Mouratoglou, G. Giannakoulas, A. Kallifatidis et al., "Left ventricular end diastolic eccentricity index predicts mortality in pulmonary arterial hypertension," European Heart Journal, vol. 35, 72 pages, 2014.

[20] R. Farooq and M. A. Malik, "On some eccentricity based topological indices of nanostar dendrimers," Optoelectronics and Advanced Materials, Rapid Communications, vol. 9, no. 56, pp. 842-849, 2015.

[21] R. Farooq, N. Nazir, M. Ali Malik, and M. Arfan, "Eccentricity based topological indices of a hetrofunctional dendrimer," Journal of Optoelectronics and Advanced Materials, vol. 17, no. 11-12, pp. 1799-1807, 2015.

[22] A. W. McCrary, J. R. Malowitz, C. P. Hornick et al., "Differences in eccentricity index and systolic-diastolic ratio in extremely low-birth-weight infants with bronchopulmonary dysplasia at risk of pulmonary hypertension," American Journal of Perinatology, vol. 33, no. 1, pp. 57-62, 2015.

[23] S. Abraham and C. G. Weismann, "Left ventricular end-systolic eccentricity index for assessment of pulmonary hypertension in infants," Echocardiography, vol. 33, no. 6, pp. 910-915, 2016.

[24] Y. Yamasaki, M. Nagao, T. Kamitani et al., "Clinical impact of left ventricular eccentricity index using cardiac MRI in assessment of right ventricular hemodynamics and myocardial fibrosis in congenital heart disease," European Radiology, vol. 26, no. 10, pp. 3617-3625, 2016.

[25] J. L. Guirao and M. T. de Bustos, "Dynamics of pseudoradioactive chemical products via sampling theory," Journal of Mathematical Chemistry, vol. 50, no. 2, pp. 374-378, 2012.

[26] D. Dimitrov, "Efficient computation of trees with minimal atom-bond connectivity index," Applied Mathematics and Computation, vol. 224, pp. 663-670, 2013.

[27] D. Dimitrov, "On structural properties of trees with minimal atom-bond connectivity index," Discrete Applied Mathematics. The Journal of Combinatorial Algorithms, Informatics and Computational Sciences, vol. 172, pp. 28-44, 2014.

[28] D. Dimitrov, "On structural properties of trees with minimal atom-bond connectivity index II: bounds on B-1- and B-2branches," Discrete Applied Mathematics, vol. 204, pp. 90-116, 2016.

[29] M. B. Ahmadi, D. Dimitrov, I. Gutman, and S. A. Hosseini, "Disproving a conjecture on trees with minimal atom-bond connectivity index," MATCH. Communications in Mathematical and in Computer Chemistry, vol. 72, no. 3, pp. 685-698, 2014.

[30] M. T. El-Sayed, S. Suzen, N. Altanlar, K. Ohlsen, and A. Hilgeroth, "Discovery of bisindolyl-substituted cycloalkane-anellated indoles as novel class of antibacterial agents against S. aureus and MRSA," Bioorganic and Medicinal Chemistry Letters, vol. 26, no. 1, pp. 218-221, 2016.

[31] A. Vásquez-Espinal, J. J. Torres-Vega, L. Alvarez-Thon, P. Fuentealba, R. Islas, and W. Tiznado, "Boron avoids cycloalkane-like structures in the LinBnH2n series," New Journal of Chemistry, vol. 40, no. 3, pp. 2007-2013, 2016.

[32] J. Caroen, A. Clemmen, J. Kámán et al., “Solid-phase synthesis of 6,7-cycloalkane-fused 1,4-diazepane-2,5-diones via a cyclization/release strategy," Tetrahedron, vol. 72, no. 1, pp. 148-160, 2016. 
[33] A. Denicourt-Nowicki, A. Lebedeva, C. Bellini, and A. Roucoux, "Highly selective cycloalkane oxidation in water with ruthenium nanoparticles," Chem CatChem, vol. 8, no. 2, pp. 357362, 2016.

[34] M. I. El-Gamal, D. Baek, and C.-H. Oh, "A new series of cycloalkane-fused coumarin sulfonates: Synthesis and in vitro antiproliferative screening," Bulletin of the Korean Chemical Society, vol. 37, no. 2, pp. 184-191, 2016.

[35] M. T. El-Sayed, K. A. Mahmoud, F. W. Heinemann, and A. Hilgeroth, "Novel tetraindoles and unexpected cycloalkane indoles from the reaction of indoles and aliphatic dialdehydes," Journal of Heterocyclic Chemistry, vol. 54, no. 1, pp. 714-719, 2017.

[36] L. Lepori, P. Gianni, and E. Matteoli, “Thermodynamic study of tetrachloromethane or heptane + cycloalkane mixtures: Excess and solvation Gibbs energies," Journal of Thermal Analysis and Calorimetry, vol. 124, no. 3, pp. 1497-1509, 2016.

[37] H. Nambu, N. Ono, W. Hirota, M. Fukumoto, and T. Yakura, "An efficient method for the synthesis of $2^{\prime}, 3^{\prime}$-nonsubstituted cycloalkane-1,3-dione-2-spirocyclopropanes using (2-bromoethyl)-diphenylsulfonium trifluoromethanesulfonate," Chemical and Pharmaceutical Bulletin, vol. 64, no. 12, pp. 1763-1768, 2016.

[38] A. Lazić, N. Trišović, L. Radovanović et al., “Towards understanding intermolecular interactions in hydantoin derivatives: the case of cycloalkane-5-spirohydantoins tethered with a halogenated benzyl moiety," CrystEngComm, vol. 19, no. 3, pp. 469483, 2017.

[39] F. E. M. Alaoui, F. Aguilar, M. J. González-Fernández, M. Dakkach, and E. A. Montero, "Excess enthalpies of ternary mixtures of (oxygenated additives + aromatic hydrocarbon) mixtures in fuels and bio-fuels: (Dibutyl-ether + 1-propanol + benzene), or toluene, at $\mathrm{T}=(298.15$ and 313.15) K," Journal of Chemical Thermodynamics, vol. 85, pp. 26-34, 2015.

[40] B. Pi-Boleda, M. Sans, M. Campos et al., "Studies on cycloalkane-based bisamide organogelators: a new example of stochastic chiral symmetry-breaking induced by sonication," Chemistry-A European Journal, vol. 23, no. 14, pp. 3357-3365, 2017. 

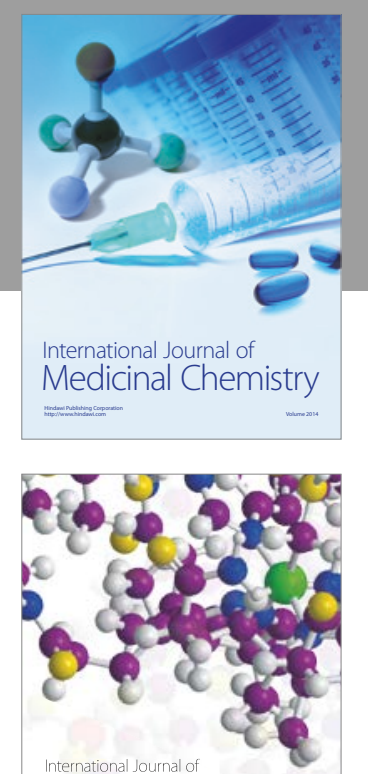

Carbohydrate Chemistry

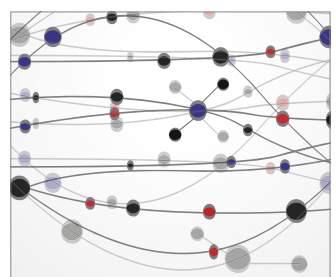

The Scientific World Journal
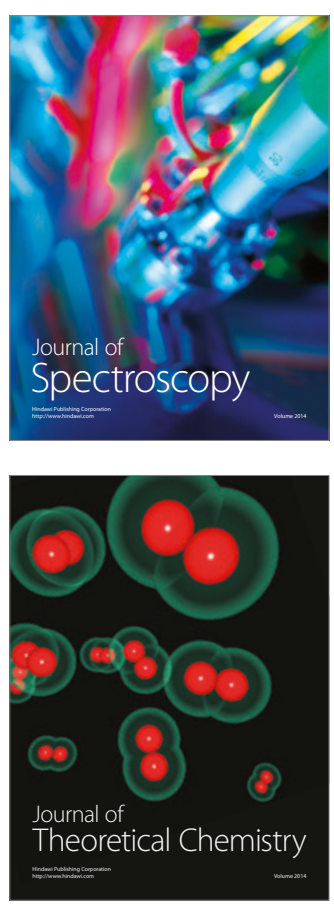
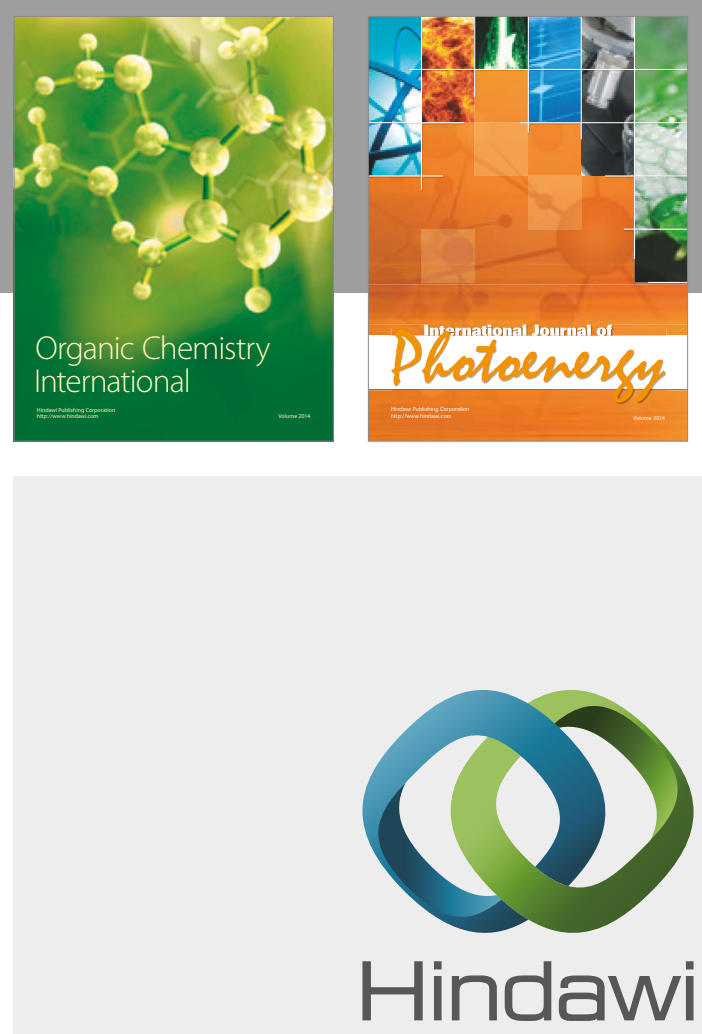

Submit your manuscripts at

https://www.hindawi.com

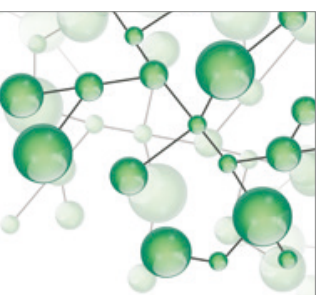

International Journal of

Inorganic Chemistry

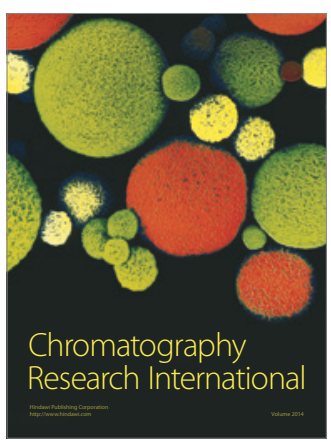

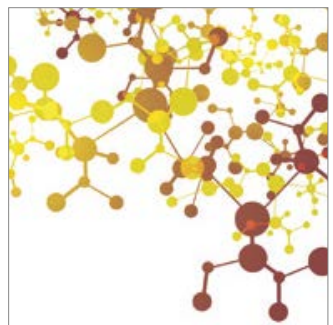

Applied Chemistry
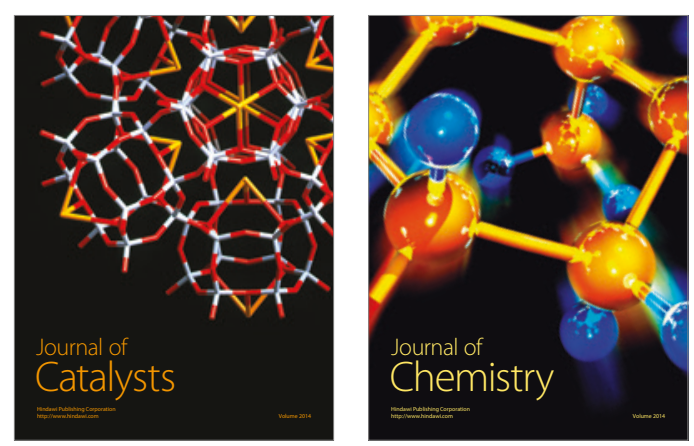
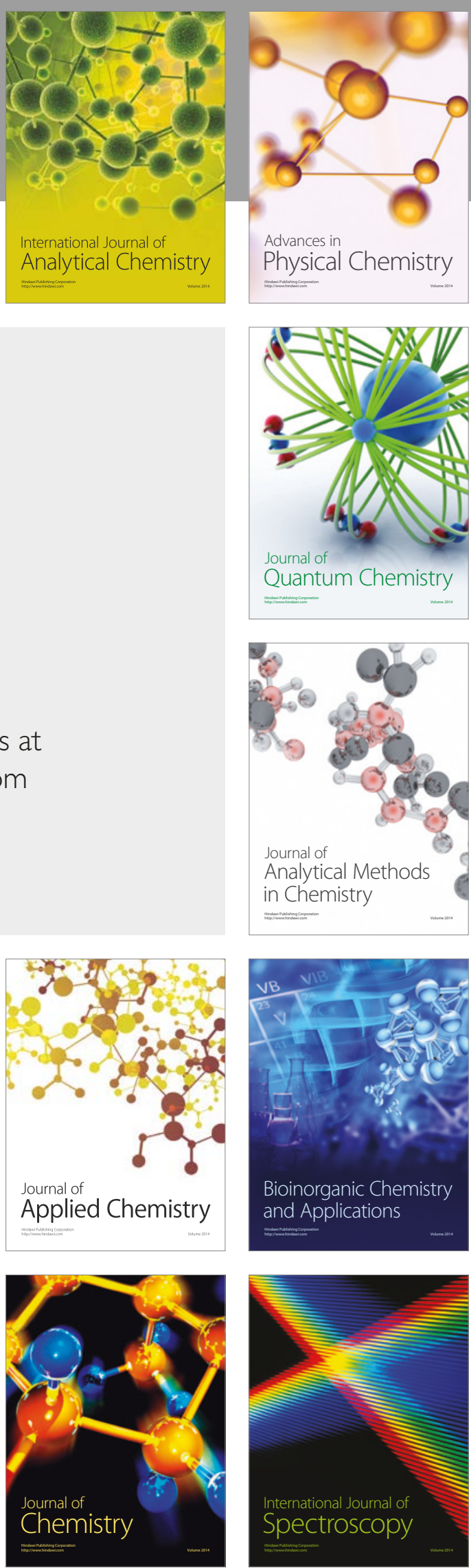